\title{
Towards improving short-term sea ice predictability using deformation observations
}

\author{
Anton Korosov ${ }^{1}$, Pierre Rampal ${ }^{2,1}$, Yue Ying ${ }^{1}$, Einar Ólason $^{1}$, and Timothy Williams ${ }^{1}$ \\ ${ }^{1}$ Nansen Environmental and Remote Sensing Center, Jahnebakken 3, Bergen, 5007, Norway \\ ${ }^{2}$ CNRS, Institut de Géophysique de l'Environnement, Grenoble, France
}

Correspondence: Anton Korosov (anton.korosov@nersc.no)

\begin{abstract}
.
Short-term sea ice predictability is challenging due to the lack of constraints on ice deformation features (open leads and ridges) at kilometre scale. Deformation observations capture these small-scale features and have the potential to improve the predictability. A new method for assimilation of satellite-derived sea ice deformation into the neXt generation Sea Ice Model (neXtSIM) is presented. Ice deformation provided by the Copernicus Marine Environmental Monitoring Service is computed from sea ice drift derived from Synthetic Aperture Radar at a spatio-temporal resolution of $10 \mathrm{~km}$ and 24 hours. We show that high values of ice deformation can be interpreted as reduced ice concentration and increased ice damage - scalar variables of neXtSIM. The proof-of-concept assimilation scheme uses a data nudging approach and deterministic forecasting with one member. Assimilation and forecasting experiments are run on example observations from January 2021 and show improvement of neXtSIM skills to predict sea ice deformation in 3-5 days horizon. It is demonstrated that neXtSIM is also capable of extrapolating the assimilated information in space - gaps in spatially discontinuous satellite observations of deformation are filled with a realistic pattern of ice cracks, confirmed by later satellite observations. The experiments also indicate that reduction in sea ice concentration plays a bigger role in improving ice deformation forecast on synoptic scales. Limitations and usefulness of the proposed assimilation approach are discussed in a context of ensemble forecasts. Pathways to estimate intrinsic predictability of sea ice deformation are proposed.
\end{abstract}

\section{Introduction}

Sea ice in the Arctic is continuously drifting and deforming under the influence of atmospheric winds and ocean currents (Sverdrup, 1950; Colony and Thorndike, 1984; Rampal et al., 2009). In summer, when ice concentration is low and ice extent is small, the sea ice is in free drift — the speed and direction of the drift are dictated only by the atmospheric and ocean drag forces and by the Coriolis force. In contrast, in winter the sea ice covers almost the entire Arctic ocean and its adjacent seas, forming a rigid and nearly continuous solid plate. As a consequence, sea ice does not drift freely anymore, but instead 
exhibits an intermittent and localised drift resulting from complex dynamics driven by a brittle mechanical behaviour. Under external forcing the ice deforms primarily as an elastic material. Internal stresses may accumulate in the material until a failure criterion is reached, which corresponds to a limit when sea ice fractures and starts deforming along the multiple narrow and elongated cracks formed, and does so until these later refreeze. Location, density and orientation of these cracks greatly control the overall and individual motion of the resulting ice pieces, from small floes $(\sim 10 \mathrm{~m})$ to large plates $(\sim 100 \mathrm{~km})$.

Under divergent ice motion these cracks become open leads, significantly increasing ocean-air heat and mass exchange and modifying local atmospheric boundary layer and ocean mixed layer. Open leads are also key both for marine fauna survival, and for facilitating ship navigation. Under convergent or shear motions sea ice ridges are formed along the cracks. Ridge sails and keels affect the drag by winds and currents. At the same time ridged ice significantly impedes navigation in the Arctic (Lindsay and Stern, 2003).

Given the importance of sea-ice fracturing for air-sea-ice interface processes, marine life and navigation, its accurate monitoring and forecasting is in great demand. Observations of cracks can be performed using satellite remote sensing by retrieval of high-resolution sea ice drift from Synthetic Aperture Radar (SAR) data and computation of sea ice deformation components (Kwok et al., 1990). The Radarsat Geophysical Processor System (RGPS) dataset was the first attempt to systematically observe sea ice deformation on high spatial resolution $(10 \mathrm{~km})$ and with high frequency (3 days) over a long period of time (winters 1996-2016) (Kwok, 1998). An operational SAR-based sea ice drift and deformation product is currently provided by the Copernicus Marine Environmental Services (CMEMS) (Saldo, 2020). This product is derived from Sentinel-1 C-band SAR data and has 12 hours frequency and $10 \mathrm{~km}$ spatial resolution. The cracks appear on satellite-derived ice deformation products as narrow (10 - $30 \mathrm{~km}$ ) and long (up to $1000 \mathrm{~km}$ ) lineaments and are also called linear kinematic features (LKFs) (Kwok, 2001).

Correct simulation and forecasting of sea ice dynamics remains a challenge. In a recent model intercomparison paper (Bouchat et al., 2021), only one model, neXtSIM (neXt Generation Sea Ice Model, Bouillon and Rampal, 2015a; Rampal et al., 2016), proved to be capable when run at the same spatial resolution as the available observations (i.e. $\sim 10 \mathrm{~km}$ ) to simulate the observed probability distribution, spatial distribution and fractal properties of sea ice deformation. Although the scaling properties of deformation are simulated correctly (Rampal et al., 2019) and the distribution of cracks looks very realistic (Olason et al., 2022), the exact timing and position of strong deformation zones, or LKFs, is not yet predicted precisely. The primary goal of our research is to improve skill in predicting LKFs by assimilating novel satellite observations of sea ice deformation. Mohammadi-Aragh et al. (2018) evaluated the potential for predicting LKFs using an ensemble of sea ice models all using a viscous-plastic rheology, but did not support their findings by observations. Our secondary goal is to quantify the actual predictability of LKFs by the neXtSIM model when combined with satellite observations via data assimilation (DA), and study factors affecting it.

Several methodological and technical challenges with assimilating sea ice deformation into a model are worth mentioning here. First, the ice deformation itself is not a model prognostic variable, so the assimilation scheme needs to perform a crossvariable update from deformation to sea ice model variables. There is also no guarantee that updated model variables will remain accurate during a forecast. For example, the ice drift is a model variable, but it is strongly dependent on external forcing 
and increments from assimilation will only survive a short period of time. Second, the observed cracks are very localized in space and time, which pose challenges in modeling its covariance structure for data assimilation methods such as 3DVar (Lorenc, 1986). Ensemble Kalman filter (EnKF) (Evensen, 2003) is potentially a good solution through estimating a flowdependent covariance structure from an ensemble of model runs. However, the current ensemble DA framework for neXtSIM (Cheng et al., 2020) is not ready to assimilate deformation yet. Therefore, and as a proof of concept, we present here a first attempt to assimilate sea ice deformation into neXtSIM using a simple nudging scheme, and perform a sensitivity analysis useful for demonstration of the approach.

The concept of sea ice deformation assimilation is presented in Section 2, followed by a detailed description of satellite observations of deformation and the methodology for assimilation and running forecasting experiments in Section 3 . The results are presented and discussed in Sections 4 and 5.

\section{Link between observed ice deformation and model state}

The central idea in our assimilation approach is that the ice in the model should become weaker - in a mechanical sense where high deformation is observed. In the current context, we simulate sea ice "weakness" evolution according to the Brittle Bingham-Maxwell (BBM) rheology (see (Olason et al., 2022) for details on how this rheology is implemented into neXtSIM). BBM belongs to a family of brittle rheologies, with earlier variations being the Elasto-Brittle (EB) (Girard et al., 2011) and the Maxwell Elasto-Brittle (MEB) (Dansereau et al., 2016). Two regimes are distinguished in the BBM: the undamaged pack ice can have small elastic (reversible) deformations; in the cracks the deformation is visco-elastic (partly permanent and partly reversible) and can become quite high (e.g. several percent per day over a spatial scale of about $10 \mathrm{~km}$ ). The BBM stress evolution equation writes as follows:

$\dot{\sigma}=E \mathbf{K}: \dot{\varepsilon}-\frac{\sigma}{\lambda}\left(1+\widetilde{P}+\frac{\lambda \dot{d}}{1-d}\right)$,

where $\sigma$ is the internal stress tensor, $E$ is the ice elasticity, $K: \dot{\varepsilon}$ is the stiffness tensor, $\lambda=\eta / E$ is the viscous relaxation time, $\widetilde{P}$ is a generalised friction term, $d$ is the ice damage.

$$
\begin{aligned}
& E=E_{0}(1-d) e^{-C(1-A)} \\
& \eta=\eta_{0}(1-d)^{\alpha} e^{-\alpha C(1-A)}
\end{aligned}
$$

where $E_{0}$ and $\eta_{0}$ are the undamaged elasticity and viscosity, and $\alpha>1$ is a constant.

$\widetilde{P}$ contains the effects of the friction element and is defined as: 
$85 \widetilde{P}= \begin{cases}\frac{P_{\max }}{\sigma_{n}} & \text { for } \sigma_{n}<-P_{\max }, \\ -1 & \text { for }-P_{\max }<\sigma_{n}<0 \\ 0 & \text { for } \sigma_{n}>0 .\end{cases}$

The friction element is active when damaged ice is converging (i.e., when the normal stress $\sigma_{n}<0$ ); when $\sigma_{n}=-P_{\max }$, the frictional forces can no longer balance the convergence and the ice starts to ridge. This threshold is defined as:

$$
P_{\max }=P h^{3 / 2} e^{-C(1-A)},
$$

Eqs. 2, 3, 5 show that increasing damage $(d)$ will decrease viscosity, while decreasing concentration $A$ will both decrease viscosity and shift the threshold $P_{\max }$, so that the ice transitions from the elastic to the viscous regime and will allow larger deformations without significant increase in internal stress.

As explained in detail in Section 3.3 we use an empirical function to convert the observed deformation to model variables, so that the update can take place in the model state space. The "observed" variable $v_{o}$ (damage or concentration) is computed from observed deformation $\epsilon_{o}$ using the following experimental formulations:

$A_{o}=f_{A}\left(\varepsilon_{o}\right)$

\section{Data and Methods}

\subsection{Satellite observations of sea ice deformation}

We used the sea ice drift and deformation dataset from CMEMS (Saldo, 2020) acquired in January 2021. The dataset comprises gridded products derived from Sentinel-1 synthetic aperture radar (SAR) images, with $10 \mathrm{~km}$ spatial resolution and 12 hours frequency. The spatial coverage of the product is irregular - the East Siberian, Laptev and Kara sea and the polar gap (north of $87^{\circ} \mathrm{N}$ ) are never covered, while other Arctic regions are observed nearly every day.

\subsection{Simulation experiments setup}

The model is run using a 900 seconds time step on a triangular mesh with $10 \mathrm{~km}$ spatial resolution covering the Arctic ocean and adjacent seas north of $65^{\circ} \mathrm{N}$. The model is forced with the European Centre for Medium-Range Weather Forecasts (ECMWF) operational analysis (Zuo et al., 2019) atmospheric forcing fields (wind speeds, air temperature, precipitation, humidity) and the TOPAZ4 (Sakov et al., 2012) ocean forcing fields (currents, sea surface temperature, sea surface salinity). 
https://doi.org/10.5194/tc-2022-46

Preprint. Discussion started: 22 February 2022

(c) Author(s) 2022. CC BY 4.0 License.

(c) (i)

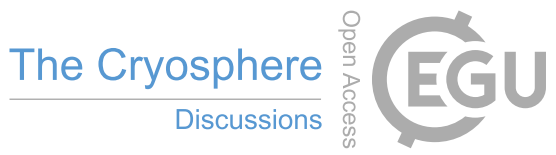

The model is initialised on 1 December 2020 from TOPAZ4 sea ice concentration, thickness, snow thickness and is run for two months. Other state variables (damage, temperature, etc) are set to a constant value. After a 30-day spin-up the concentration, thickness and deformation fields reach an equilibrium and the period from 1st to 31st Jan 2021 is used for analysis (see scheme on Fig. 1). The forecast runs are initialised every day in January 2021 from the assimilated damage and concentration variables and other variables from the free run. Forecasts are produced every day for a period of 5 days.

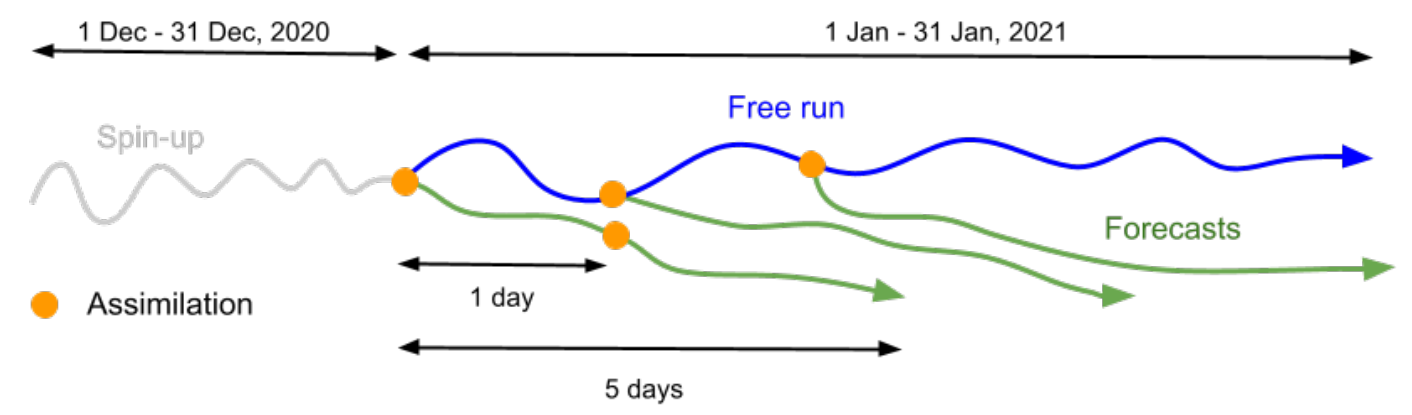

Figure 1. Scheme of the spin up run (gray curve), free run (blue curve) and forecasts (green curves). Time scale is not preserved.

For initialising a forecast run at time $t_{0}$ from assimilated damage and concentration, the deformation is taken from satellite observations of drift between times $t_{0}$ and $t_{1}$ separated by 24 hours (see scheme in Fig. 2). For evaluation, the deformation is computed from the forecasted drift between two dates separated by 24 hours (e.g., $t_{0}$ and $t_{1}$, or $t_{1}$ and $t_{2}$ ) and compared with observed deformation corresponding to the same time period. Observations (and corresponding evaluations) between $t_{0}$ and $t_{1}$ are hereafter denoted as having zero lead time. The deformation components (divergence, shear, vorticity, total, denoted as $\left.\varepsilon_{d i v}, \varepsilon_{s h r}, \varepsilon_{v o r}, \varepsilon_{t o t}\right)$ are computed from the simulated drift using contour integrals of velocities over the triangular elements of the model's mesh as explained in the previous work of (e.g. (Bouillon and Rampal, 2015b)).

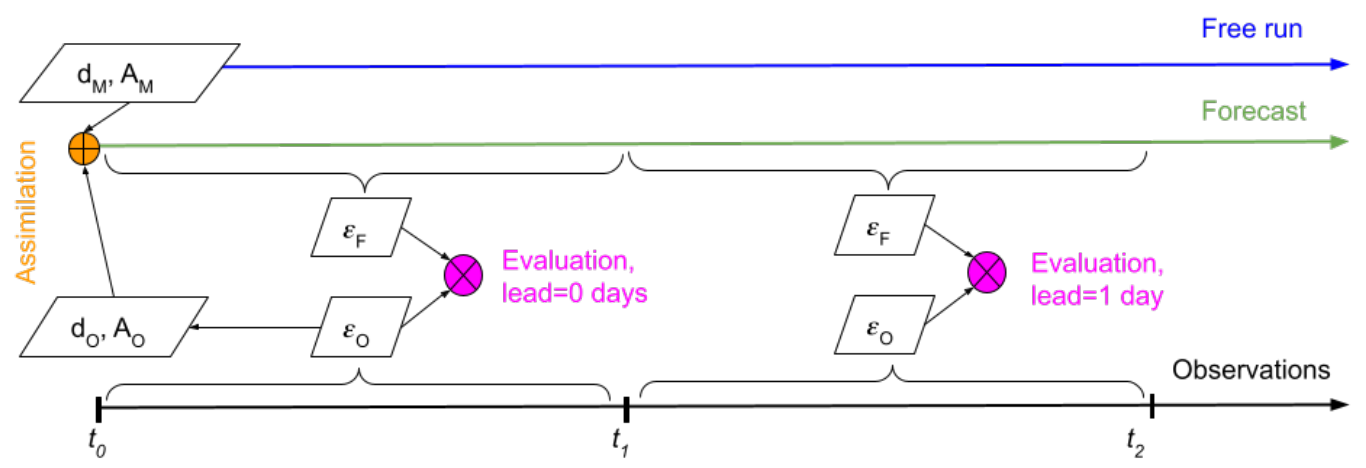

Figure 2. Scheme of assimilation (orange circle) and evaluation (purple circle) of forecasts (green arrow) using observations (black arrow). Eps, $\mathrm{d}$ and A denote deformation, damage and concentration correspondingly. Subscripts $\mathrm{F}$ and $\mathrm{O}$ denote forecast and observation. 
https://doi.org/10.5194/tc-2022-46

Preprint. Discussion started: 22 February 2022

(c) Author(s) 2022. CC BY 4.0 License.

(c) (i)

\subsection{Data assimilation method}

The function $f_{d}($ ) (Eq. 6 in Section 2) is established from the following considerations. A true relationship between deformation and model variables is multivariate and involves nonlinear dependencies on the external forcing: even fully-damaged ice will not deform without winds or currents. Satellite observations and our previous studies with the neXtSIM model show that the values of ice deformation follow a log-normal distribution (Marsan et al., 2004; Rampal et al., 2019). As detailed in the supplementary materials (see Appendix I) our simulations show that a log-normal function can accurately describe the distribution of $(1-d)$. Damage's distribution has a mean about 0.95 and exhibits a short tail towards 1 and a relatively longer tail towards smaller values. Our simulations also show a linear relationship between damage and total deformation in log-log space, i.e.:

$\log _{10}\left(k_{1}+1-d\right)=k_{2}+k_{3} \log _{10}\left(\varepsilon_{t o t}\right)$

where $k_{2}$ and $k_{3}$ are linear regression coefficients and $k_{1}$ is a small offset to prevent damage getting too close to 1 (a value that damage should never reach in progressive damage models; Amitrano et al., 1999). The expression for computing damage is thus:

$d=f_{d}\left(\varepsilon_{t o t}\right)=1-10^{k_{2}+k_{3} \log _{10}\left(\varepsilon_{t o t}\right)}-k_{1}$

The coefficients $k_{1}, k_{2}$ and $k_{3}$ are found experimentally following two steps (see Appendix I for details). First, both the model damage $\left(d_{M}\right)$ and deformation $\left(\varepsilon_{M}\right)$ are taken from the free run and the preliminary coefficient values are found using the least squares method. Second, the deformation is taken from the satellite observations $\left(\varepsilon_{O}\right)$ and damage is computed using the preliminary coefficients $d_{O}=f_{d}\left(\varepsilon_{O} ; k_{1}, k_{2}, k_{3}\right)$. Then the frequency distributions of the model damage $\left(d_{M}\right)$ and the reconstructed damage $\left(d_{O}\right)$ are compared and the coefficients $k_{1}, k_{2}$ and $k_{3}$ are updated by fitting these distributions. The latter step is required due to the initial differences existing between the simulated and observed frequency distributions of deformation that result from varying integration time of satellite observations, noise in observations, and uncertainties in simulated drift.

The function $f_{A}()$ in Eq. 7 in our assimilation scheme has a simpler form:

$A=f_{A}\left(\varepsilon_{t o t}\right)=1-a_{1} \varepsilon_{t o t}$

It can be justified by the fact that in case of pure divergence the decrease in concentration is a product of divergence rate $\left(\varepsilon_{d i v}\right)$ and time, therefore the coefficient $a_{1}$ has a meaning of integration time. However in Eq. 10 we compute deformation from $\varepsilon_{t o t}$ assuming that ice breaks and should become weaker also in case of convergence or shear. The coefficient $a_{1}$ is found empirically through the sensitivity experiments described below. 
https://doi.org/10.5194/tc-2022-46

Preprint. Discussion started: 22 February 2022

(c) Author(s) 2022. CC BY 4.0 License.

(c) (i)

We update the damage and concentration variables in the model according to the observed deformation using a simple leastsquares nudging approach as a proof of concept for DA. The updated state variable is computed as a weighted average of the forecasted variable $\left(v_{m}\right)$ and the variable computed from the observed deformation $\left(v_{o}\right)$ :

$v_{a}=w * v_{o}+(1-w) v_{m}$

where $w$ is the weight applied to observations. As defined here, the weight can be interpreted as precision (inverse of uncertainty) of observation relative to the model variable. In variational assimilation schemes the uncertainties are characterised by error co-variance matrices, while here we assume no correlation structure between variables and only characterise the relative precision (signal to noise ratio of observation-to-model variable error variances) as a single weight. Though simplified, we allow this weight to be individually specified for different variables and spatially varying, therefore giving flexibility to the update scheme. Also, we note that we assume the model variables are spatially uncorrelated, and that the variable on each model mesh point can be updated independently. Considering that sea ice deformation is accommodated along nearly 1D geometrical features (i.e. fractures), the very small spatial correlation approximation is reasonable.

We parameterize the weights as:

$w=w_{v} W$

where $w_{v}$ is a variable specific weight (either $w_{d}$ or $w_{A}$ ) and $W$ is weight dependent on observed deformation:

$W= \begin{cases}1, & \text { if } \varepsilon>\varepsilon_{\text {min }}, \\ 0, & \text { otherwise }\end{cases}$

where $\varepsilon_{\min }$ is a threshold for total deformation.

It is known that low values of deformation have higher uncertainty (Dierking et al., 2020), so it is sensible to update model variables only when the observed deformation exceeds the threshold value. This threshold localizes the impact of assimilating observed deformation to only be effective in the vicinity of ice cracks.

The variable-dependency is tested by setting the weight as 0 or 1 for damage and concentration (i.e., letting assimilation update only damage or concentration, or both to see the impact of the update).

\subsection{Sensitivity experiments}

The list of parameters tested in the sensitivity experiments is provided in Table 1. Since it was difficult to distinguish between the individual impacts of $w_{v}$ and $W$ in Eq. 12, only values of 0 and 1 were tested for $w_{d}$ and $w_{A}$, thus enabling or disabling assimilation of damage and concentration in the experiments. The values for $a_{1}$ and $\varepsilon_{\min }$ were tested within reasonable ranges, i.e. expected decrease of concentration due to ice deformation given the observable ranges of deformation.

Over 30 experiments were run following the algorithm:

- Choose assimilation parameters from a predefined space and save in a configuration file 
https://doi.org/10.5194/tc-2022-46

Preprint. Discussion started: 22 February 2022

(c) Author(s) 2022. CC BY 4.0 License.

(c) (i)

Table 1. Tested parameters of sea ice deformation assimilation scheme

\begin{tabular}{l|l|c|c} 
Parameter & Description & Eq. & Tested values \\
\hline$a_{1}$ & Coefficient for computing sea ice concentration from deformation & 10 & $0.1,0.3,0.5,0.9,1.2,1.5,2$ \\
$w_{d}$ & Weight of damage assimilation & 12 & 0,1 \\
$w_{A}$ & Weight of concentration assimilation & 12 & 0,1 \\
$\varepsilon_{\min }$ & Threshold of total deformation for applying assimilation & 13 & $0.01,0.02,0.1$
\end{tabular}

- Run series of 31 forecasts in January 2021 with these parameters

- Evaluate each forecast by comparing simulated and observed deformations

- Average the evaluated quality metrics over the month

The effect of assimilation on the prediction skill is evaluated by comparison of the simulated and observed deformation fields. As mentioned above, ice deformation is related to the processes of ridging and lead opening and, therefore by using the deformation as the reference we evaluate the model skills to correctly predict leads and ridges - crucial information for safe navigation, ecological and climate studies.

The forecasts were evaluated using two quality metrics: area of maximum cross-correlation (MCC) and difference in $90^{\text {th }}$ percentile, hereafter referred to as $A_{M C C}$ and $D_{P 90}$. The cross-correlation (see Korosov and Rampal, 2017, for explanation) between the observed and simulated deformation was computed in a sliding window of $20 \mathrm{x} 20$ pixels (200 x $200 \mathrm{~km})$ and the maximum value from the cross-correlation matrix was preserved for each floating window (see Fig. 3 for an example of MCC). The area where the MCC is above 0.35 normalised to the total area of available satellite observations was considered as one quality metric for one deformation field's forecast. This metric indicates the level of correspondence to observations at a relatively fine spatial scale ( $1-2$ pixels, $10-20 \mathrm{~km})$.

For calculating the other quality metrics the $90^{\text {th }}$ percentile was computed from observed and simulated deformations in the same sliding window. Then the root mean square difference of P90 values is computed from the entire deformation field in order to quantify the match between the model and satellite observations at the basin scale.

\section{Results}

\subsection{Impact of assimilation on deformation fields}

The impact of assimilation is demonstrated by a comparison of sea ice deformation maps from observations on $22^{\text {nd }}$ January 2021 (Fig. 3.A), a free run without assimilation (Fig 3.B) and a forecast with assimilation of concentration computed from deformation (Fig 3.C). In that example only concentration was updated with $a_{1}=1.2, w_{A}=1$, and $\varepsilon_{\min }=0.02$. Other assimilation parameters are tested below in Section 4.2. Fig. 3 clearly shows that the field of deformation predicted in the free run (Fig 3.B) is different from the observations both in terms of location, sharpness and orientation of cracks, as well as in terms 
https://doi.org/10.5194/tc-2022-46

Preprint. Discussion started: 22 February 2022

(C) Author(s) 2022. CC BY 4.0 License.

(c) (i)

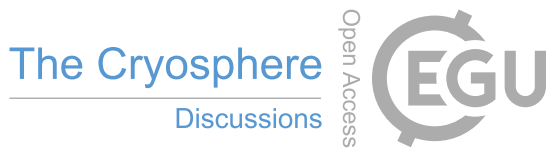

of the deformation magnitude. The corresponding maximum cross correlation map (Fig 3.C) shows very low values (average correlation is 0.2 ) and the map of P90 difference (Fig 3.D) confirms strong underestimation of total deformation (mostly $<-0.1$ $\left.\mathrm{d}^{-1}\right)$.

After assimilation the field of deformation changes substantially where the observations were present (such area on Fig 205 3.C is highlighted). Visually the position of cracks correspond well to the observations, which is supported by high values of correlation (average correlation is 0.7, Fig. 3.F) and low difference of P90 (average $D_{P 90}$ is $0.01 \mathrm{~d}^{-1}$, Fig. 3.G). We also note that the deformation field changes outside the area of assimilation (semi-transparent regions on Fig. 3.B and 3.C). For example, cracks near the North Pole change orientation by 45 degrees, the cracks north-east of the New Siberian Islands (next to the observations) disappear, many features in the Beaufort sea reduce intensity or disappear. Thus, due to its rheology, neXtSIM is able to extrapolate and create realistic connections between the observed and assimilated pieces of LKFs. 
https://doi.org/10.5194/tc-2022-46

Preprint. Discussion started: 22 February 2022

(c) Author(s) 2022. CC BY 4.0 License.

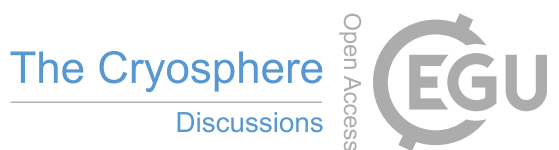
(c) (i)

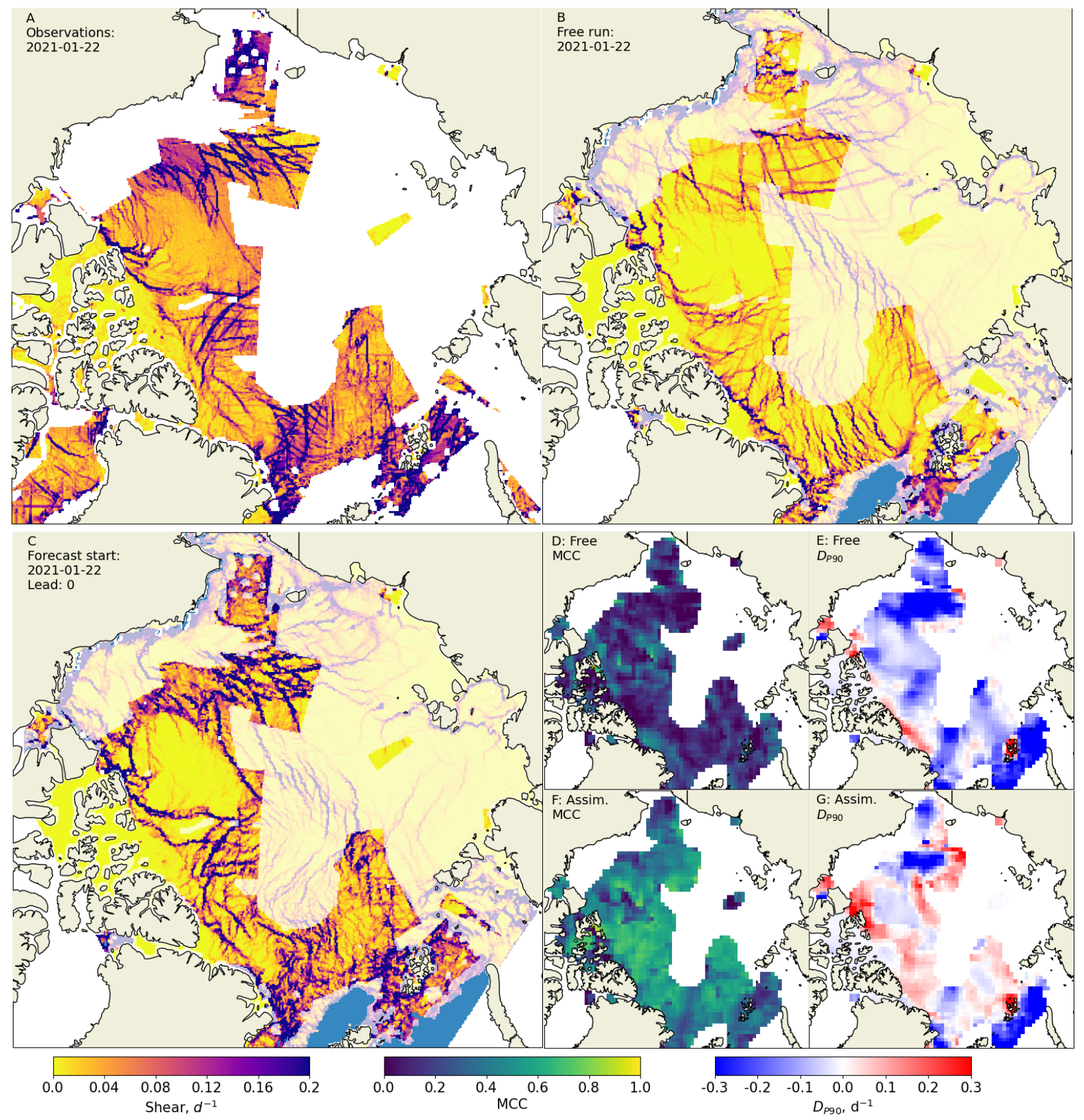

Figure 3. Maps of sea ice total deformation showing impact of assimilation. A - observations on 22 January 2021. B - free run. C - forecast with assimilation of deformation with zero days lead time. D - map of maximum cross correlation of the free run and observations. E - map of difference between P90 of the free run and observations. F and G are maps of MCC and $D_{P 90}$ for the forecast with assimilation. Colobars below are given for deformation, MCC and $D_{P 90}$. 
https://doi.org/10.5194/tc-2022-46

Preprint. Discussion started: 22 February 2022

(c) Author(s) 2022. CC BY 4.0 License.

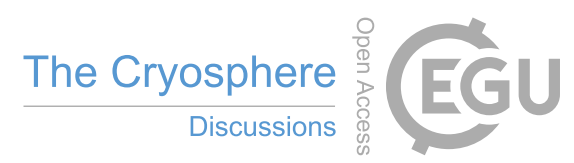

On the second day of forecast the difference between the observed and simulated deformation fields generally increases. Fig. 4 zooms on a smaller region $(1500 \times 1500 \mathrm{~km})$ in the central Arctic and shows that in some areas the spatial correlation between deformation fields decreases (dark blue arrows on Fig. 4.F), whereas in other areas it stays high (green arrows on Fig. 4.F). This example was chosen to demonstrate that the deformations extrapolated by the model out of the region with assimilated data may compare well with observations on the next day of forecast (red circle on Fig. 4.F), but sometimes may become different (yellow circle on Fig. 4.F).
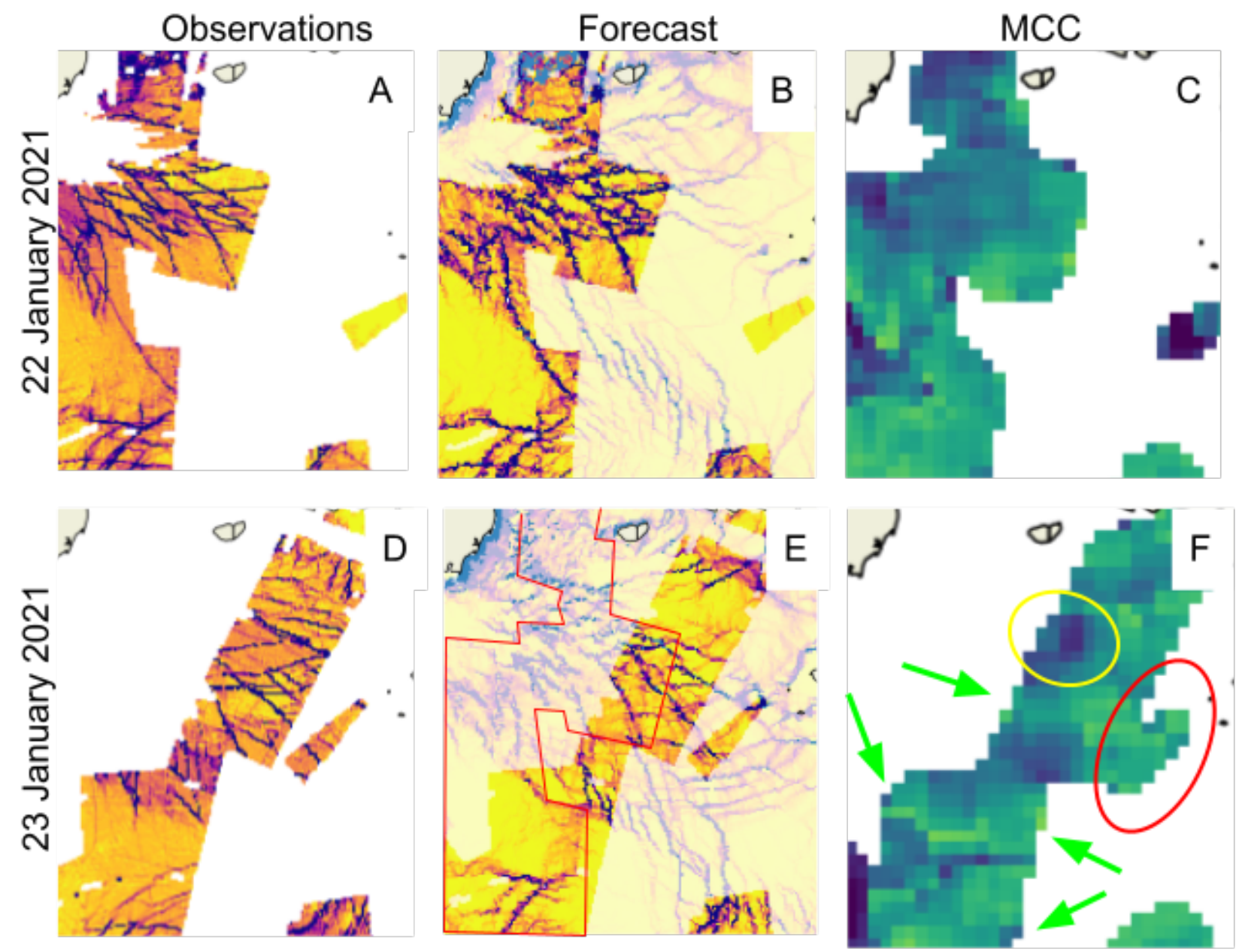

Figure 4. Maps of deformation on observations (A, D) and forecasts (B, E) for 22 January (A, B) and for 23 January (D, E). Maps on C and $\mathrm{F}$ show maximum cross correlation computed in the sliding window. Red polygon on E shows extent of observations on the previous day. Green arrows on F show where MCC stays high. Yellow and red circles on F show where extrapolated deformation is low or high, correspondingly. Colobars are the same as on Fig. 3. 
The evaluation metrics $A_{M C C}$ and $D_{P 90}$ were computed for each day of the 31 forecasts as well as for a free run and for the persistent forecast. In the persistence forecast the analysis from the first day is compared to observations from consecutive days. The metrics were averaged and plotted against lead time as shown on Fig. 5. Comparison of the metrics shows that the free run deformations are quite different from the observations - only $\sim 10 \%$ of the area has sufficiently high correlation and the difference in deformation P90 is above 0.13 day $^{-1}$. Assimilation substantially improves the correspondence to the observations: area covered by valid deformation exceeds $80 \%$ and $D_{P 90}$ drops to 0.06 days $^{-1}$. On the second day the area with correlated deformation drops to $30 \%$ and with lead time longer than 3 days it is almost indistinguishable from the free run. The difference of P90 does not increase that fast - it remains lower than the free run during the entire 5-day forecast. The error in the persistence forecast grows faster than in the dynamic forecast - by the fifth day the high correlation area is lower by $5 \%$ and the $D_{P()}$ is larger by 0.3 day $^{-1}$.

These results indicate that assimilation of sea ice deformation has an impact over a period as long as 5 days. The exact position of the cracks is improved only during the first $1-1.5$ days, but the pan-Arctic spatial distribution is improved over longer time scales.
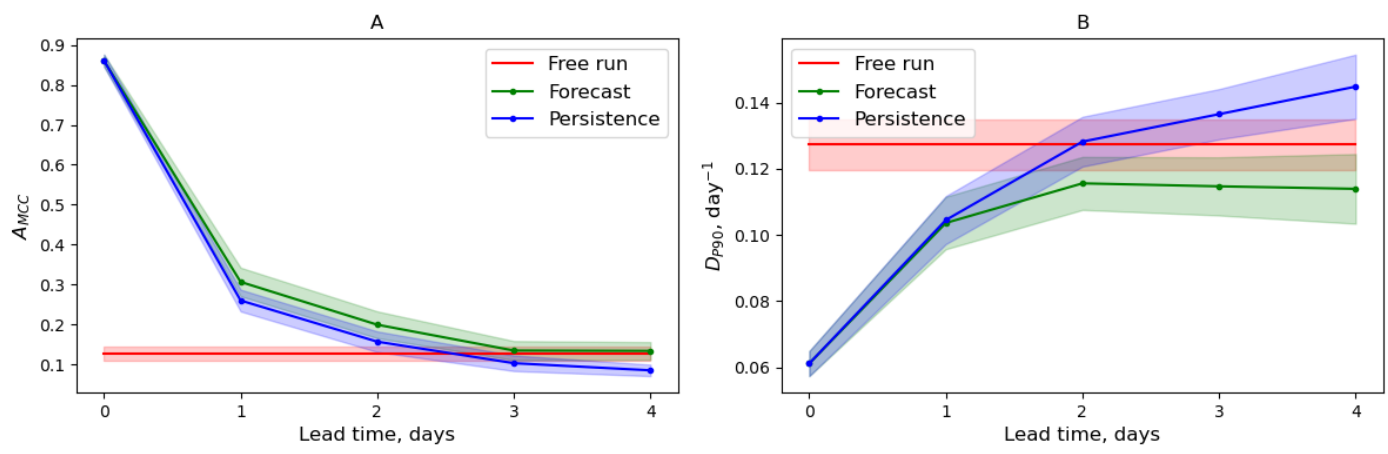

Figure 5. Evolution of the area with high correlations (A) and root mean square difference (B) averaged over 31 days for the forecasts (green), persistence (blue) and the free run (red). Filled region shows one quarter of standard deviation.

\subsection{Sensitivity to assimilation parameters}

The sensitivity experiments with the $a_{1}$ parameter of our assimilation scheme show that during the first day of forecast the minimum $D_{P 90}$ ( 0.06) and maximum $A_{M C C}$ ( 0.8 ) are observed when $a_{1}$ values are in the range of -1.5 - -0.9 (see Fig. 6). A value of $a_{1}=-1$ corresponds to linear reduction in concentration due to pure divergence, therefore for sufficient effect of assimilation the concentration needs to be decreased slightly more when computed from the total deformation.

With higher $a_{1}$ the error is higher both on the first and on the consequent days of forecasts (up to the level of free run, $D_{P 90}$ $=0.12, A_{M C C}=0.15$ ), indicating that the impact of assimilation is too weak. With higher $a_{1}$, the error is higher on the first forecast day but is close to the minimum on the second and third days. That demonstrates the impact of excessive concentration nudging, which exaggerates deformations on the first day but keeps the information on cracks for a longer time. 
Several experiments with $\varepsilon_{\text {min }}$ show that even very spatially localized assimilation, when $\varepsilon_{\min }=0.1$, quite considerably impacts the field of deformations: the quality is only slightly lower than in the forecasts with $\varepsilon_{\min }=0.02\left(D_{P 90}\right.$ higher by 0.1 , $A_{M C C}$ lower by 0.1$)$.

The experiments with $w_{d}$ cannot detect a strong impact of damage assimilation. With $w_{d}=1$, the quality is only marginally better: $D_{P 90}$ lower by $0.01, A_{M C C}$ higher by 0.01$)$.
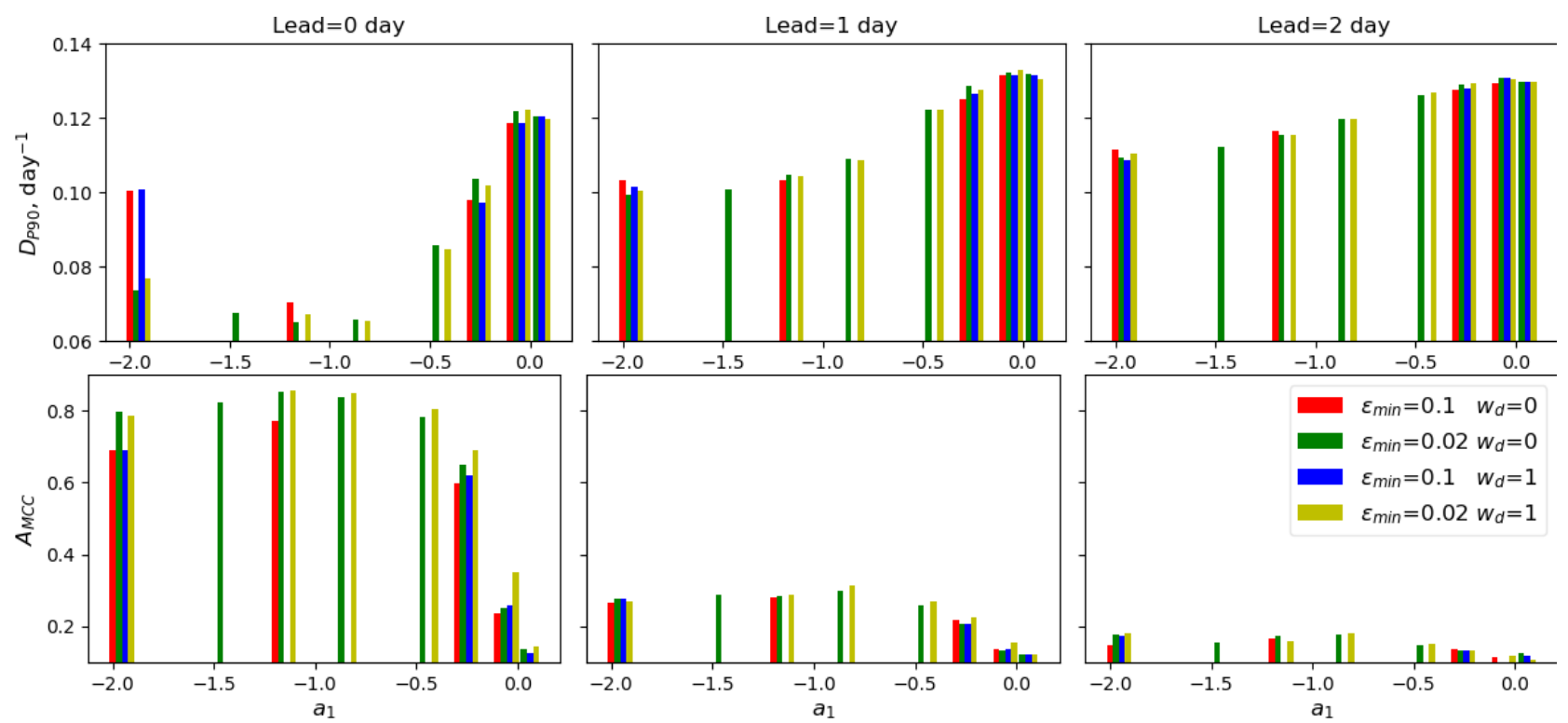

Figure 6. Dependence of the averaged error metrics $\left(D_{P 90}\right.$ and $\left.A_{M C C}\right)$ on parameters of assimilation. Red: $\varepsilon_{m i n}=0.1$ (localized impact), $w_{d}=0$; Green: $\varepsilon_{\min }=0.02$ (wide impact), $w_{d}=0$; Blue: $\varepsilon_{\min }=0.1$ (localized impact), $w_{d}=1$; Yellow: $\varepsilon_{\min }=0.02\left(\right.$ wide impact), $w_{d}=$ 1. Bars with $a_{1}=0$ illustrate experiments with damage assimilation only.

\section{Discussions}

\subsection{Theoretical and practical usefulness}

We present the first successful attempt to use the observed sea ice deformation to improve sea ice model prediction skills on a horizon of 3-5 days. The approach we used to update the model fields is relatively simple - the simulated values of sea ice concentration and damage are nudged towards the concentration and damage computed from the observed ice deformation. In addition, we use this simplified nudging scheme with only a few tuning parameters, instead of using more sophisticated DA methods such as the EnKF, for the sake of confirming several hypotheses and providing a proof of concept. First, it demonstrates in practice that information contained in the deformation fields can be related to the model state variables. Second, it shows the time scales at which the forecast of deformation can be improved by updating some model variables. Third, it proves that even if nudging is spatially limited by observations (or even very localized in high deformation zones) it corrects the deformation 
pattern simulated with the neXtSIM model in the entire basin. Finally, it reveals the relative importance of the assimilation parameters (e.g., $a_{1}$ vs. $\varepsilon_{\min }$ ) and, as explained below, the relative importance of the model state variables.

The experiments, in which we minimized the difference between simulation and observations by tuning the parameters in a grid search, can be interpreted as an optimization of the DA hyperparameters. These parameters can be associated with uncertainties in observed deformation, which are either spatially constant $\left(a_{1}, w_{c}\right.$ and $\left.w_{d}\right)$, or spatially varying $\left(\varepsilon_{\min }\right)$. These uncertainties can be related to the diagonal terms in the error covariance matrices used in more sophisticated EnKF and 4DVar methods. However, the uncertainty of the model concentration and damage is either not known or not taken into account. Further study is needed to derive a full covariance matrix, especially the off-diagonal terms depicting cross-variable relations. Knowledge of this obvious weakness in the presented approach paves the road for the planning of future experiments: an ensemble of neXtSIM runs (with perturbed forcing) should be used for evaluating uncertainties in the model variables; detection of covariance between the observed deformation and the model state (not restricting to damage and concentration); and eventually updating the model state using state-of-the-art DA techniques.

From the practical point of view, the presented approach is useful for the current realisation of the forecasting platform neXtSIM-F. neXTSIM-F is currently used operationally for providing sea ice forecasts through CMEMS. For now, the platform can only operate with a single neXtSIM member but is outfitted with full functionality required for operational work: download of forcing and observations data, assimilation of sea ice concentration and type, running of the model, visualisation and validation of the forecasts. Integration of the suggested assimilation approach into neXtSIM-F will improve forecasts of sea ice leads and ridges, providing information that is crucial for tactical navigation in the Arctic.

\subsection{Impact of damage and concentration assimilation}

As indicated in Olason et al. (2022), neXtSIM is a damage propagation sea ice model and damage is used for changing elasticity and viscosity. So why can't we see the impact of damage assimilation in our experiments? We believe there are two major reasons for that. First, the damage is acting in the model at much smaller timescales than our observations of sea ice deformation. Damage can increase from 0 to 1 in just a few model steps before it eventually starts to decay due to a mechanical healing mechanism. The increase of damage takes only a few minutes of simulated time, during which apparent sea ice elasticity and viscosity are proportionally decreased and large-scale and permanent deformation is allowed, accompanied by sea ice internal stresses relaxation. The available observations of deformation are taken on time scales of 24 hours and cannot detect such rapid processes.

The second reason is that the BBM rheology assumes the ice to be a two-phase material. One phase is that of small deformations, permitted by increasing damage, and the other is of large deformations, permitted by decreasing concentration. Damage linearly impacts the ice stiffness, whereas the concentration acts in the exponent (see Eqs. 2 and 3). In a compact ice cover the ice can only deform by first damaging. If this deformation is mainly convergent the concentration stays high and the deformations remain small. If, on the other hand the flow diverges, concentration drops and the deformations can become large. 
These considerations can be illustrated by looking at what happens in two distinct elements that we picked up from the free run from the model mesh at different locations of the integration domain (see Fig. 7). In the first element (blue lines on Fig. 7) the normal and tangent stresses grow until they reach the Mohr-Coulomb envelope, when the damage starts to build up (we are interested in the second event). When damage reaches 0.75 (indicated by the blue dashed line) a small jump in the total deformation is observed and the stresses are relaxed on the next step. However the deformation was not large enough to sufficiently decrease the concentration and therefore the later remains close to $100 \%$. This means that even if the damage reaches high values in this particular mesh element, the deformation remains low. In the second element (orange lines on Fig. 7) the initial deformation at the break-up event is larger, the concentration decreases rapidly and, as a result, the deformation on later steps reaches much higher values.
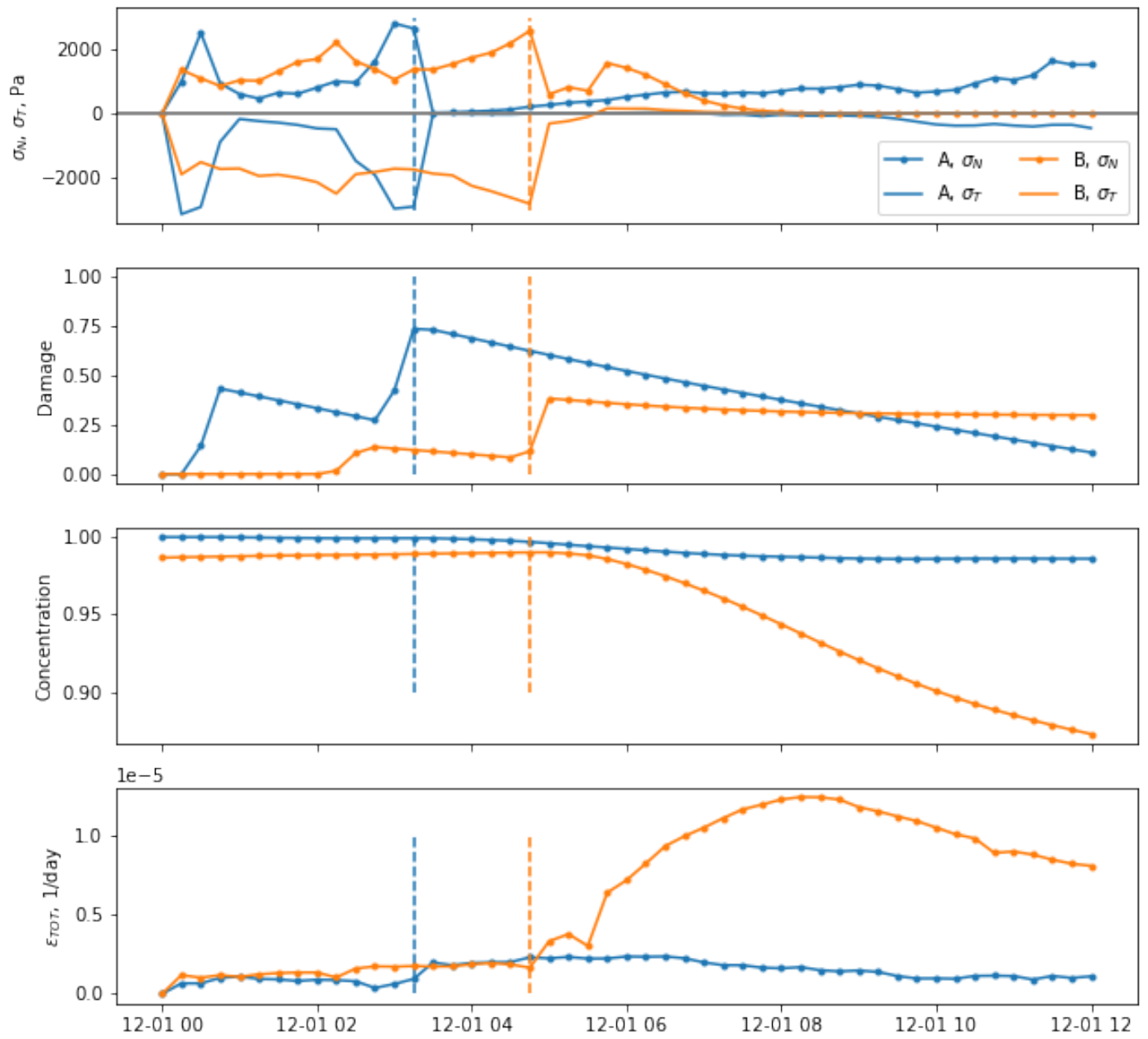

Figure 7. Evolution of internal stress $\left(\sigma_{N}\right.$ and $\left.\sigma_{T}\right)$, damage $(d)$, concentration $(A)$ and total deformation $\left(\varepsilon_{\text {tot }}\right)$ in two independent elements (blue and orange lines). Vertical lines show approximately the time of ice break-up. 
https://doi.org/10.5194/tc-2022-46

Preprint. Discussion started: 22 February 2022

(c) Author(s) 2022. CC BY 4.0 License.

(c) (i)

\subsection{Towards evaluation of short-term sea ice predictability}

How predictable sea ice features are at kilometre and daily time scale still remains an open question. Mohammadi-Aragh et al. (2018) gives a first estimate of the potential predictability of LKFs to be 4-8 days using MITgcm ensemble runs perturbed with atmospheric conditions from the ECMWF Ensemble Prediction System. They also found that additional perturbations in sea ice thickness initial conditions do not contribute significantly to the forecast error growth in LKFs. The current study provides a new predictability estimate in a different context. Our results show that the deterministic forecast of LKFs gains prediction skill for 2-5 days after assimilating deformation observations, indicating a clear impact of improving accuracy of sea ice initial conditions. The viscous-plastic (VP) rheology used in MITgcm is known to have a less realistic slower time evolution of LKFs (Hutter et al., 2018) than the BBM rheology in neXtSIM (Olason et al., 2022). As a result, the sea ice simulated by the BBM rheology has more rapid error growth (loses skill faster) due to the correctly resolved intermittent ice motion and localised ice deformation.

In real world application, the prediction skill of sea ice LKFs depends on several sources of uncertainties/deficiencies in the system (listed below), further studies are needed to address each of them and build a complete picture of the current prediction skill of sea ice at daily time scales and the room for future improvements.

- Uncertainties in atmospheric and ocean forcing. Accuracy of contemporary weather and oceans forecasts is quite high (Zhang et al., 2019; Xie et al., 2017). Nevertheless, forcing the ice model with slightly inaccurate wind fields or ocean currents may only slightly change the ice drift pattern, but the ice deformation, being a spatial derivative, will be affected more. Surface wind variability is an important source of sea ice uncertainties (Rabatel et al., 2018; Cheng et al., 2020). A recent study showed that increasing the accuracy (resolution) of atmospheric boundary condition will improve the representation of sea ice extreme breakup events in the neXtSIM during passage of polar cyclones (Rheinlaender et al. in review). More comprehensive studies are needed to evaluate the impact of external forcing uncertainties on sea ice LKF forecasts at daily time scales.

- Rheology and model parameterization. Uncertainties in rheology parameters were shown to be another error source for sea ice forecasts (Urrego-Blanco et al., 2016; Cheng et al., 2020). The BBM rheology (Olason et al., 2022) was implemented in neXtSIM quite recently to replace the previous Maxwell Elasto-Brittle rheology of Dansereau et al. (2016). It was only calibrated to be compared to statistical properties of sea ice deformation derived from the RGPS observation dataset (Kwok et al., 1990) and on large-scale sea ice thickness and drift time series. Therefore, it is not tuned for predicting the exact position of cracks in the sea ice cover, which may impact the predictability we obtain in this study. The BBM rheology can be further tuned and compared to the modified Elasto-Visco-Plastic rheology (mEVP, Bouillon et al., 2009) that is already an available option in neXtSIM (Olason et al., 2022) for estimating the impact of rheology on the sea ice predictability.

- Model numerics. In neXtSIM, the model equations are derived and solved on a triangular mesh that deforms with the ice motion in a pure-Lagrangian approach. In addition to the physics of the rheological model, this Lagrangian approach may 
contribute to improving the localisation of cracks in space and time. However, in such framework a remeshing procedure is used when the mesh becomes too distorted in order to replace too skewed triangles with nearly isosceles triangles. After the remeshing procedure, the model variables are interpolated from the old to the new mesh using a conservative interpolation via supermesh construction. This results in a diffusion of the model fields and likely impacts the prediction skill of the model. Ongoing work of implementing the BBM rheology in an Eulerian version of the neXtSIM model, using a Discontinuous Galerkin advection scheme, will allow us to study the impact of the use of a fixed Eulerian grid compared to a Lagrangian mesh on the efficiency of the data assimilation method and sea ice deformation predictability.

- Initial conditions for sea ice states. The impact from initial condition uncertainties can be revisited using the neXtSIM with the new BBM rheology. Future studies can run ensembles of neXtSIM simulations with perturbation of ice thickness, concentration, damage, ice types and the ice thickness distribution to assess propagation of errors across variables and scales and evaluate their impact on predictability.

- Observing network and data assimilation In practice, the choice of DA method and availability of observations will also impact the accuracy of initial conditions and therefore impact the predictability. In this study, we made a first attempt to assimilate deformation derived from the operationally available ice drift product from CMEMS, which provides information at the smaller daily time scales for sea ice features. Future studies can assess how observations on different scales (e.g. with higher spatial and temporal resolution) impact the predictability. Also, DA performance can be further improved in future studies using more sophisticated methods to further improve the accuracy in initial conditions.

\section{Conclusions}

The presented method for assimilation of satellite-derived sea ice deformation into the neXt generation Sea Ice Model (neXtSIM) efficiently ingests information about where the ice is mechanically weak and improves forecasts of ice deformation for a horizon of 3-5 days. Despite using a relatively simple nudging approach, neXtSIM is capable of extrapolating the spatially discontinuous satellite observations of deformation by connecting the elements of linear kinematic features in a realistic manner. The main idea behind the proposed method is to relate local sea ice weakness to local reduced ice concentration and increased ice damage, which are computed as functions of observed ice deformation. Experiments with the parameters of the DA scheme show that updating concentration substantially improves neXtSIM skills on the synoptic scale, whereas updating damage has an effect only on time scales of a few hours, which is difficult to confirm by satellite observations. It is anticipated that update of the ice damage with more frequent observations will play a bigger role in increasing the accuracy of the short range forecasts to fully take advantage of the brittle rheology. The presented approach can already be used in operational forecasting systems for improving deterministic forecasts, or it can be developed further and integrated into a variational assimilation approach based on ensemble runs. 
https://doi.org/10.5194/tc-2022-46

Preprint. Discussion started: 22 February 2022

(C) Author(s) 2022. CC BY 4.0 License.

(c) (i)

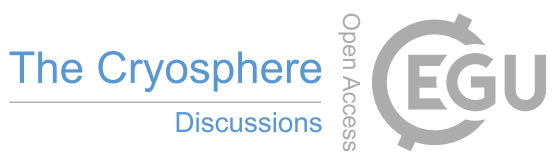

Data availability. Sea ice deformation data is publicly available at the Copernicus Marine Environmental Cervices portal: https://resources. marine.copernicus.eu/product-detail/SEAICE_GLO_SEAICE_L4_NRT_OBSERVATIONS_011_006/.

Author contributions. This work is the follow up of an original idea of PR. AK and PR designed the methodology and AK carried out the experiments and the analyses. EO, TW, PR and AK developed the neXtSIM model code and AK performed the simulations. AK prepared the manuscript with contributions from all co-authors.

Competing interests. The authors declare that they have no conflict of interest.

365 Acknowledgements. This study was supported by the following projects: ImpSim funded by the Service hydrographique et océanographique de la marine (SHOM), MOIRA funded by the European Space Agency (ref.no. 4000129593/19/I-DT), and MUSIC funded by the Norwegian Research Council (ref.no. 325292 - FORSKER21). We thank to the Copernicus Marine Environmental Cervices for providing the sea ice drift and deformation data product. We also thank Sylvain Bouillon for the numerous discussions that lead to the idea developed in this study. 
https://doi.org/10.5194/tc-2022-46

Preprint. Discussion started: 22 February 2022

(c) Author(s) 2022. CC BY 4.0 License.

(c) (i)

\section{References}

Amitrano, D., Grasso, J. R., and Hantz, D.: From diffuse to localised damage through elastic interaction, Geophys. Res. Lett., 26, 2109-2112, 1999.

Bouchat, A., Hutter, N. C., Chanut, J., Dupont, F., Dukhovskoy, D. S., Garric, G., Lee, Y. J., Lemieux, J.-F., Lique, C., Losch, M., and et al.: Sea Ice Rheology Experiment (SIREx), Part I: Scaling and statistical properties of sea-ice deformation fields, Earth and Space Science Open Archive, p. 36, https://doi.org/10.1002/essoar.10507397.1, 2021.

Bouillon, S. and Rampal, P.: Presentation of the dynamical core of neXtSIM, a new sea ice model, Ocean Modelling, 91, 23-37, https://doi.org/10.1016/j.ocemod.2015.04.005, 2015a.

Bouillon, S. and Rampal, P.: On producing sea ice deformation data sets from SAR-derived sea ice motion, The Cryosphere, 9, 663-673, https://doi.org/10.5194/tc-9-663-2015, 2015b.

Bouillon, S., Ángel Morales Maqueda, M., Legat, V., and Fichefet, T.: An elastic-viscous-plastic sea ice model formulated on Arakawa B and C grids, Ocean Modelling, 27, 174-184, https://doi.org/10.1016/j.ocemod.2009.01.004, 2009.

Cheng, S., Aydoğdu, A., Rampal, P., Carrassi, A., and Bertino, L.: Probabilistic Forecasts of Sea Ice Trajectories in the Arctic: Impact of Uncertainties in Surface Wind and Ice Cohesion, Oceans, 1, 326-342, https://doi.org/10.3390/oceans1040022, 2020.

Colony, R. and Thorndike, A. S.: An estimate of the mean field of Arctic sea ice motion, Journal of Geophysical Research, 89, 10623, https://doi.org/10.1029/JC089iC06p10623, 1984.

Dansereau, V., Weiss, J., Saramito, P., and Lattes, P.: A Maxwell elasto-brittle rheology for sea ice modelling, The Cryosphere, 10, 1339$1359,2016$.

Dierking, W., Stern, H. L., and Hutchings, J. K.: Estimating statistical errors in retrievals of ice velocity and deformation parameters from satellite images and buoy arrays, The Cryosphere, 14, 2999-3016, https://doi.org/10.5194/tc-14-2999-2020, 2020.

Evensen, G.: The Ensemble Kalman Filter: theoretical formulation and practical implementation, Ocean Dynamics, 53, 343-367, https://doi.org/10.1007/s10236-003-0036-9, 2003.

Girard, L., Bouillon, S., Weiss, J., Amitrano, D., Fichefet, T., and Legat, V.: A new modelling framework for sea ice mechanics based on elasto-brittle rheology, Ann. Glaciol., 52(57), 123-132, 2011.

Hutter, N., Losch, M., and Menemenlis, D.: Scaling Properties of Arctic Sea Ice Deformation in a High-Resolution Viscous-Plastic Sea Ice Model and in Satellite Observations, Journal of Geophysical Research: Oceans, 123, 672-687, https://doi.org/10.1002/2017JC013119, 2018.

Korosov, A. and Rampal, P.: A Combination of Feature Tracking and Pattern Matching with Optimal Parametrization for Sea Ice Drift Retrieval from SAR Data, Remote Sensing, 9, 258, https://doi.org/10.3390/rs9030258, 2017.

Kwok, R.: The RADARSAT Geophysical Processor System, https://doi.org/10.1007/978-3-642-60282-5_11, 1998.

Kwok, R.: Deformation of the Arctic Ocean sea ice cover between November 1996 and April 1997: a qualitative survey, Solid Mechanics And Its Applications, 94, 315-322, 2001.

Kwok, R., Curlander, J., McConnell, R., and Pang, S.: An ice-motion tracking system at the Alaska SAR facility, IEEE Journal of Oceanic Engineering, 15, 44-54, https://doi.org/10.1109/48.46835, 1990.

Lindsay, R. W. and Stern, H. L.: The RADARSAT Geophysical Processor System: Quality of Sea Ice Trajectory and Deformation Estimates, Journal of Atmospheric and Oceanic Technology, 20, 1333-1347, https://doi.org/10.1175/1520- 
https://doi.org/10.5194/tc-2022-46

Preprint. Discussion started: 22 February 2022

(c) Author(s) 2022. CC BY 4.0 License.

(c) (i)

Lorenc, A. C.: Analysis methods for numerical weather prediction, Quarterly Journal of the Royal Meteorological Society, 112, 1177-1194, https://doi.org/10.1002/qj.49711247414, 1986.

Marsan, D., Stern, H., Lindsay, R., and Weiss, J.: Scale Dependence and Localization of the Deformation of Arctic Sea Ice, Physical Review Letters, 93, 178 501, https://doi.org/10.1103/PhysRevLett.93.178501, 2004.

410 Mohammadi-Aragh, M., Goessling, H. F., Losch, M., Hutter, N., and Jung, T.: Predictability of Arctic sea ice on weather time scales, Scientific Reports, 8, 6514, https://doi.org/10.1038/s41598-018-24660-0, 2018.

Olason, E., Boutin, G., Korosov, A., Rampal, P., Williams, T., Kimmritz, M., Dansereau, V., and Samake, A.: A new brittle rheology and numerical framework for large-scale sea-ice models, in review, Journal of Advances in Modeling Earth Systems, 0, 0, https://doi.org/https://doi.org/0, 2022.

415 Rabatel, M., Rampal, P., Carrassi, A., Bertino, L., and Jones, C. K. R. T.: Impact of rheology on probabilistic forecasts of sea ice trajectories: application for search and rescue operations in the Arctic, The Cryosphere, 12, 935-953, https://doi.org/10.5194/tc-12-935-2018, 2018.

Rampal, P., Weiss, J., and Marsan, D.: Positive trend in the mean speed and deformation rate of Arctic sea ice, 1979-2007, Journal of Geophysical Research, 114, C05 013, 2009.

Rampal, P., Bouillon, S., Ólason, E., and Morlighem, M.: NeXtSIM: A new Lagrangian sea ice model, Cryosphere, 10, 1055-1073, https://doi.org/10.5194/tc-10-1055-2016, 2016.

Rampal, P., Dansereau, V., Olason, E., Bouillon, S., Williams, T., Korosov, A., and Samaké, A.: On the multi-fractal scaling properties of sea ice deformation, The Cryosphere, 13, 2457-2474, https://doi.org/10.5194/tc-13-2457-2019, 2019.

Sakov, P., Counillon, F., Bertino, L., Lisæter, K. A., Oke, P., and Korablev, A.: TOPAZ4: An ocean sea ice data assimilation system for the North Atlantic and Arctic, Ocean Sci., 8, 633-662, 2012.

Saldo, R.: Global Ocean - High Resolution SAR Sea Ice Drift, https://resources.marine.copernicus.eu/product-detail/SEAICE_GLO_ SEAICE_L4_NRT_OBSERVATIONS_011_006/INFORMATION, https://doi.org/10.48670/moi-00135, 2020.

Sverdrup, H. U.: Physical Oceanography of the North Polar Sea, Arctic, 3, 178-186, 1950.

Urrego-Blanco, J. R., Urban, N. M., Hunke, E. C., Turner, A. K., and Jeffery, N.: Uncertainty quantification and global sensitivity analysis of the Los Alamos sea ice model, Journal of Geophysical Research: Oceans, 121, 2709-2732, 2016.

430 Xie, J., Bertino, L., Counillon, F., Lisæter, K. A., and Sakov, P.: Quality assessment of the TOPAZ4 reanalysis in the Arctic over the period 1991-2013, Ocean Science, 13, 123-144, https://doi.org/10.5194/os-13-123-2017, 2017.

Zhang, F., Sun, Y. Q., Magnusson, L., Buizza, R., Lin, S.-J., Chen, J.-H., and Emanuel, K.: What Is the Predictability Limit of Midlatitude Weather?, Journal of the Atmospheric Sciences, 76, 1077-1091, https://doi.org/10.1175/JAS-D-18-0269.1, 2019.

Zuo, H., Balmaseda, M. A., Tietsche, S., Mogensen, K., and Mayer, M.: The ECMWF operational ensemble reanalysis-analysis system for ocean and sea ice: a description of the system and assessment, Ocean Science, 15, 779-808, https://doi.org/10.5194/os-15-779-2019, 2019. 